\title{
ADOPTION OF SOCIAL MEDIA AND MANAGEMENT OF LESSONS LEARNED IN IT PROJECT
}

\author{
Chomchai Lertamornrat
}

Suan Sunandha Rajabhat University, Bangkok, Thailand

This research explores the gap in the management of lessons learned (MLL) in an Information Technology project (IT), validating a model (named Target) with the support of wiki platform in a medium-sized company in the IT industry. This model supports the following MLL processes: awareness, collection, verification, storage, dissemination and reuse. This study adopts the paradigm of the Design Science Research and the Technical Action Research method to instantiate the Target model in the implementation phase of an IT project. The practical implications can be seen in the company's learning to introduce the $M L L$, improve design productivity, increase employee collaboration and better disseminate knowledge.

Keywords: knowledge management; lessons learned; web 2.0; information technology projects; social media; DSR; TAR

\section{Introduction}

Project environment, best practices, and management of lessons learned (MLL) have received attention in practice and research for more than a decade (Hartmann et al., 2014).

The MLL issues are faced by companies that must to overcome modern challenges in a structured way (Wiewiórą et al., 2015). Projects by libraries with plans, budgets, learned reports and lessons, operationalizing the MLL (Chronéer et al., 2015) can improve the distribution of knowledge in a social network (Crawford et al., 2005; Milton, 2010).

More recent studies focus on mechanisms to improve knowledge sharing (Gomes et al., 2016), based on practical communities, exploiting social media (Lee et al., 2015). In fact, the better understanding of social media role in managing LL remains a challenge to the Project Management (PM) community.

Chaves and Veronese (2014) proposed to introduce social media in the MLL. Rosa, Chaves, Oliveira, and Pedron (2016) created an LL model named Target, supported by social media, which includes LL processes throughout the project life cycle.

The use of social media facilitates shared real-time communication and is already a reality. On the other hand, the practice suggests that censures imposed by organizations in communication determine business rules, and are poorly understood by most project

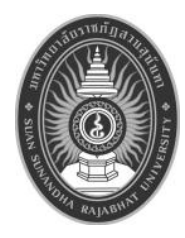

Chomchai Lertamornrat

lecturer in College of Innovation and management, Suan Sunandha Rajabhat University,

Bangkok, Thailand, member of evaluation team

Research interests: IT, Innovative business, information communications, virtual organization, global market of innovations

E-mail: chomchai_le@ssru.ac.th 
managers (Levitt, 2011). Adding to this scenario, the management of projects, such as in the software industry, begins to incorporate the Project Management 2.0 approach (Kerzner, 2015), including it in its practical storage and processing in the cloud, which suggests the need to evolve in training, and other practices (Levitt, 2011). In this context, Rosa et al. (2016) created an LL model named Target, supported by social media, which includes LL processes throughout the project life cycle.

This research aims to contribute to the MLL by an instantiation of the Target model using a wiki. The Target model, which was validated by experts in projects (Rosa et al., 2016), is now applied in practice to foster innovation in the MLL. The model proposes the adoption of awareness, collection, verification, storage, dissemination and reuse processes in MLL.

The following research question underpins this study: to what extent does the Target model contribute to MLL in an IT project? The research has two goals: to validate the instantiation of Target model in an IT project using a wiki; and obtain from users their perception of ease and convenience of use, and assess possible behavioral changes in MLL.

\section{Theoretical Foundation}

The approach of organizational knowledge management is characterized by the ability of a company to create knowledge as a corporate asset and understand the need to manage it and give it the same care as that devoted to the achievement of tangible assets.

The field of knowledge management generates an increasing interest in several areas such as the project management. This happens in the discipline of knowledge management because its processes and tools aim to increase the potential knowledge for making more assertive decisions, contributing to the competitive advantage of the organization. The MLL is a part of knowledge management, contributing to the identification of the problems recorded and studied an activity that helps maintain the social capital of the organization. Lessons learned (LL) projects as a learning activity, in many cases, become critical for the project in the implementation of an Information System (IS) (Milton, 2010; Park \& Lee, 2014).

The MLL may pose psychological challenges and individual characteristics that motivate people to share information (Jugdev et al., 2014).

Argote (2011) analyzes organizational learning considering three steps in its composition: creation, retention and transfer of knowledge. When companies learn from experience, new knowledge is created for the company. Knowledge must then be maintained so that it exhibits some persistence and validity over time (Argote, 2011). Knowledge can be transferred within and between project units. When transferring knowledge, the project is affected by experience, based on that of previous projects.

One of the most common ways to share project knowledge is to capture the positive and negative aspects of LL (Wiewiora et al., 2015). Thus, when used effectively, the process can assist project managers in the reuse of knowledge and prevention of future errors and their repetition (Pemsel et al., 2013). However, the processes of capture, storage, analysis and reuse of LL often remain underestimated. Wiewiora and Murpy (2015) argue that Web-based collaboration is easily accessible, intelligible and user-friendly, allowing the sharing of project knowledge to overcome existing problems. As a result of this study, some characteristics of these tools are highlighted in relation to LL: storage and sharing, visibility, 


\section{ADOPTION OF SOCIAL MEDIA AND MANAGEMENT}

feedback, priority, Capture mechanisms, capture tools, systemic approach and quality. Based on these authors, the LL can have a strong influence on people, groups and especially on the more experienced, inside and outside the projects.

In addition to these factors, the use of processes and tools, especially those with easy access, such as the collaborative tools of web 2.0, can help in the management of LL (Veronese et al., 2016). These issues contribute to the objectives of the research, during the instantiation of the Target model and in the use of the wiki, with its convenience, ease of use and behavior change, contributing to the MLL.

The increasing adoption of virtual teams in IT projects contributes to the need for employee mobility and the need for collective, rapid decision making by project team members in different locations (Reed et al., 2010). This contributes to the increase in virtual teams and the use of web 2.0 collaborative tools as support for decision making (Turban et al., 2011).

Technology plays an important role, and the use of centralized systems is especially important to support collaboration (De Mattos et al., 2015). Wikis are suitable for projects with teams physically distributed in remote locations, allowing team members to organize and publish content, freeing the project manager for greater dedication to other activities (Milton, 2010).

Wikis assist project teams in defining scope, documentation, collaboration, discussion and follow-up activities. Added to these scenarios, IT projects and software quality are very dependent on the definition of the scope, the specification of tasks, communicated and understood. This scenario contributes to the assertion that wikis can be a potential solution for defining scope and tasks effectively used to promote an evolutionary mindset throughout the project (Gholami et al., 2011; Han et al., 2007; Rosa et al, 2016). Holtzblatt et al. (2010) concluded that the return on investment depends heavily on how the technology is adopted. For a positive effect on collaboration, the authors conclude that we should consider what prevents workers from coding and sharing knowledge. It is necessary to be aware of the work environment that users are entering, checking the possibility of sharing knowledge in various tools.

Holtzblatt et al. (2010) also suggest an incentive structure, clear policies and guidelines, and support and encouragement of information sharing practices. A wiki-based system allows you to maintain and control activities that include complete control over versions and their content. Wikis also include a history of revisions, allowing archived changes to be rolled back, and the traceability of information, which helps control inappropriate information by identifying the authors through the history. This ease of control and identification does not eliminate user mistrust regarding malicious content, posing doubts about the reliability of the wiki. Added to this, security issues should be strongly considered in building the process of using the wiki (Gholami et al., 2011).

\section{Methodology}

The main criterion of quality of knowledge is its validation by the informed public scientific information based on the presentation of empirical evidence (Aken, 2005; Huff, 2000). Founded in this concept, this study introduces a model of MLL in field-testing with technology-based support, and an analysis of its use. This study stimulates the effort to improve MLL through practical situations involving the subject studied. In an exploratory 
context with practical actions, this study is supported by a qualitative and exploratory approach. The ontology of this research is objective, related to facts and data, and hence measurable. This study creates conditions to interfere in the appearance or modification of facts in order to explain what happens with practical phenomena in an MLL environment in an IT project.

The Target model is the artifact validated in practice under the paradigm of Design Science Research (DSR) (Gregor et al., 2013; Pournader et al., 2015). Recent debates seek to contribute to the practice through research, for example the European communities in Germany and the UK (Gregor et al., 2013).

The importance of research highlights the orientation of the DSR paradigm, emphasizing the link between the utility and the truth, "the truth lies in the utility" (Pournader et al., 2015). This view of DSR does not preclude its potential to contribute to the improvement of the theory (Cole et al., 2005). In line with the paradigm, the method adopted in this work was Technical Action Research (TAR). The method supports the search for a solution to a problem using an artifact or prescription, narrowing the gap between theory and practice. While most methods of empirical research seek to study the phenomenon as it is currently, researchers using action methods (e.g. action research) seek to intervene in the situations studied (Wieringa, 2012).

A satisfactory solution, but not necessarily perfect, it is a common practice in DSR (Cole et al., 2005; Huff et al., 2006; Papas et al, 2012). TAR under the same platform as the Action Research method adds to the technical nature of research, which is conducted on two simultaneous and independent fronts, the practical and the theoretical. This research proposes a solution to a specific and empirical problem, where literature does not offer a theoretical framework, a feature found in Action Research (Patton, 2015).

The research was carried out in two cycles. During the instantiation of the artifact, adjustments, actions and their evaluations were carried out between cycles.

In an attempt to find a solution for the customer's problem, meetings were held in order to understand the project mechanisms, MLL, the company's characteristics, develop the wiki pages with managers, directors and all the 29 employees. Ten employees, from three distinct areas, worked directly in the research.

The interviews began forty-five days after the wiki pages were released for use and after two months of awareness and preparation work. After the first three interviews, it was possible to make adjustments, increasing awareness actions and dissemination of the work, in addition to stimulating employee participation. During the research, documents, meeting minutes, project records and processes were collected, contributing to the researcher's analysis and observations in the field, concluding with a confirmatory focus group, two months after the instantiation of the artifact.

\section{Analysis of Results}

The analysis of the interviews, confirmatory focus group, the participation of the researcher and direct observation in the field, plus the content of the documents collected were enough to make it explicit that the company did not have a formal process of MLL. In addition, at the beginning of the study, decentralized records were found without any standardization in meeting minutes, network repositories and notebooks in desk drawers. 


\section{ADOPTION OF SOCIAL MEDIA AND MANAGEMENT}

The contributions of the participants were identified by the analysis of their wiki records containing their experiences. These records represent the practice of employees in their work. During the interviews, most respondents said that people need to make it a habit to use LL, referring to themselves and co-workers, either in the registry or the reuse of knowledge, as stated by I4:

I4 - "The habit of registering still needs to grow a lot, it is a matter of habit, culture, the company, and getting to the person that I am."

This behavioral issue was also mentioned in the confirmatory focus group, and during the observation of the researcher, which is clear in the statement by I6: I6 - "I don't use the internal tool a lot in my work directly with what I do on a day-to-day basis." It was noticed that at least one of those involved in the use of the wiki had difficulty in organizing his experience explicitly. This scenario suggests that there is difficulty in creating the habit of registering lessons learned.

The implementation of the awareness process was the main task during the field research period in the company, because it stimulates the participation of all project members. This implementation was the first intervention of the researcher, starting at the research dissemination meeting with discussion on LL. Throughout the project, we identified the need to increase awareness and dissemination of LL.

Therefore, disclosure has been expanded with the contribution of weekly internal email on the issue to all sections at weekly meetings aimed at agile software development. Also along this line the inferences throughout the day on LL, began to include reminders and encouragement by managers to use the artifact during the search. The researcher expanded the need for awareness and dissemination, based on some accounts collected in interviews:

I3 - I haven't used the process and the wiki yet to support my current activity because I'm still in training in my new function. But I consider lesson learned important and the process is very practical".

I6- "I don't use the internal tool a lot in my work directly with what I do on a day-today basis" I7 - "Even if I cannot post to the wiki, I send a general email to all staff, to formalize things, but people end up not taking any notice" .

I9 - "The customer asks for something new that no one uses, agent needs to know how to be able to give support, guide, sometimes it is not documented, there is only the information with the person who developed it".

I7 - "In my notebook there are already some processes to take up, reserve a little time to go to the wiki and create."

Most interviewee responses were very positive about awareness in the use of the artifact. Only two interviewees were the exception to the positive outcome of the process, as they state that the artefact has not reached its goal. I6 answers this question hesitantly, unsure how to explain his point:

I6 - "It needs a more mature, higher contribution of people interacting. I do not use it, I do not need it, I can get it because I know it's there, but it's easier to wait for another person to do it".

For this same purpose, four respondents cite usability as a point to be improved in the wiki. Unlike, I5 valued the artifact, the process and the tool in addition to their importance in LL: I5 - "I think the lessons learned are very useful for anyone, regardless of the area, it is a worthwhile experience, any company and people are welcome to use it. The sharing of information is valid since people can apply it in their daily routine." 
The research highlights that the educational and cultural level of those involved, added to the fact that they have an average age of 26 , are favorable as they belong to a generation involved with collaborative technologies in their daily lives. This scenario contributes to the employees' prioritization of their participation with individual concerns (Papadopoulos et al., 2013).

The research thus shows a way for using the Target model for MLL in an IT company. Target was implemented in the project execution phase, with positive evaluation for all involved in the project. The research highlights the ease and convenience of use of a wiki, contributing to MLL in a medium-sized IT company.

The data collected throughout the research by interviews, focus group, documentation and observation of the researcher suggest that the Target model was positively validated. In this context, the results of question 9 present proposals by respondents for improvements in the architecture of the wiki.

The field research in the execution phase included dynamic adaptations of the process from information captured in the context of research and implementation of the process, throughout the artifact use, based on the inference of the investigator.

\section{Contributions to Theory and Practice}

The paradigm on which the research is based, DSR, gives this research an epistemological position of pragmatism, and thus leads to the pursuit of improving both the theory and practice.

The theoretical contribution lies in the practical utility of an LL model, which was validated in the field. In addition, this research adds new knowledge to the MLL in projects by promoting innovation in the MLL and introducing a LL model validated in the IT industry.

The use of the wiki in the research contributes to the vision of Faraj, Jarvenpaa, \& Majchrzak (2011) as a tendency for interaction in companies, especially in innovation with the adoption of social media.

As a practical implication, the use of Target model brings as a contribution a better MLL in an IT project. It contributes to the construction of knowledge through action and experimentation, considering the social environment in which it is inserted, consisting of a group of people working on the project. In this sense, the instantiation of an LL model in an IT project using a wiki facilitated stakeholder understanding as to how useful this model is.

\section{Final Remarks}

The paper presented the empirical validation of an LL model in the execution of an IT project. With the active participation of the researcher in the field along with the company's professionals, observing and gathering information from the daily routines of work, it is possible to make a positive evaluation of the model's implementation.

In this sense, the wiki contributes to the collaborative environment proposed by the Target model, facilitating the exchange of experiences.

The main limitation of this research lies in the impossibility of validating the model throughout all stages of the IT project. We should also consider that the Thailand economic environment affected the research setting throughout the year 2015, since the company faced 


\section{ADOPTION OF SOCIAL MEDIA AND MANAGEMENT}

necessary staff reductions and was unable to replace employees. Further research includes the validation of the Target model in all phases of the project life cycle and in other industries.

We also suggest a quantitative study hypothesizing relationships (e.g. rising awareness through the offering of wiki training programs will lead participants to perceive the tool as more useful, which in turn leads to more usage), control variables, and moderators (e.g. years of experience). In addition, the Target model could also be instantiated with other social media.

\section{References:}

Aken, J. E. V. (2005). Management research as a design science: Articulating the research products of model 2 knowledge production in management. British Journal of Management, 16 (1); 19-36.

Argote, L. (2011). Organizational learning research: Past, present and future. Management Learning, 439-446.

Chaves, M. S., Araújo, C. C. S., Rosa, D. V, Gloria Jr, I., \& Nogueira, C. D. (2016). A new approach to managing Lessons Learned in PMBoK process groups: the Ballistic 2.0 Model. International Journal of Information Systems and Project Management, 4(1): 27-45.

Chaves, M. S. \& Veronese, G. (2014). A Proposal to Manage Lessons Learned in Projects: Web 2.0 Technologies to Promote Innovation. International Journal of Innovation, 2(1), 1-17.

Chronéer, D., \& Backlund, F. (2015). A Holistic View on Learning in Project-Based Organizations. Project Management Journal. 9.

Cole, R., Purao, S., Rossi, M., \& Sein, M. K. (2005). Being Proactive: Where Action Research meets Design Research. In: International Conference on Information Systems (ICIS). Proceedings. Paper 27.

Crawford, J., \& Cabanis-Brewin, J. (2005). Optimizing human capital with a strategic project office: select, train, measure, and reward people for organization success. In Presented at the PMI Global Congress.

De Mattos, C. A., \& Laurindo, F. J. B. (2015). Collaborative platforms for supply chain integration: Trajectory, assimilation of platforms and results. Journal of Technology Management and Innovation, 10(2), 79-92.

Faraj, S., Jarvenpaa, S. L., \& Majchrzak, A. (2011). Knowledge Collaboration in Online Communities. Organization Science, 22(5), 1224-1239.

Gholami, B., \& Murugesan, S. (2011). Global IT Project Management Using Web 2.0. International Journal of Information Technology Project Management, 2(3), 30-52.

Gomes, F.; Oliveira, M. \& Chaves, M. S. (2016). Knowledge Sharing in Project Management Process Groups. 17th European Conference on Knowledge Management, Northern Ireland, UK, 307315.

Gregor, S., \& Hevner, A. R. (2013). Positioning and Presenting Design Science Research for Maximum Impact. MIS Quarterly, 37(2), 337-355.

Han, B. M., \& Anantatmula, V. S. (2007). Knowledge sharing in large IT organizations: a case study. Gower Publishing, Ltd.., 37(4), 421-439.

Hartmann, A., \& Dorée, A. (2014). Learning between projects: More than sending messages in bottles. International Journal of Project Management. 6.

Holtzblatt, L. J., Damianos, L. E., \& Weiss, D. (2010). Factors Impeding Wiki Use in the Enterprise : A Case Study. In CHI'10 Extended Abstracts on Human Factors in Computing Systems. ACM., 3, 4661-4675.

Huff, A., Tranfield, D., \& Aken, J. E. V. (2006). Management as a Design Science Mindful of Art and Surprise: A Conversation Between Anne Huff, David Tranfield, and Joan Ernst van Aken. Journal of Management Inquiry, 15(4), 413-424. 
Jugdev, K., \& Wishart, P. (2014). Mutual caring - Resolving habituation through awareness: Supporting meaningful learning from projects. Project Management Journal, 45(2), 66-82.

Kerzner, H. R. (2015). Project Management 2.0. John Wiley \& Sons.

Lee, L., Reinicke, B., Sarkar, R., \& Anderson, R. (2015). Learning Through Interactions: Improving Project Management Through Communities of Practice. Project Management Journal, 46(1), $40-52$.

Levitt, R. E. (2011). Towards project management 2.0. Engineering Project Organization Journal. 5.

Milton, N. (2010). The Lessons Learned Handbook: Practical approaches to learning from experience. New Delhi: Oxford - Cambridge.

Papadopoulos, T., Stamati, T., \& Nopparuch, P. (2013). Exploring the determinants of knowledge sharing via employee weblogs. International Journal of Information Management, 33(1), 133146.

Papas, N., Keefe, R. M. O., Seltsikas, P., O’Keefe, R. M., \& Seltsikas, P. (2012). The action research vs design science debate: reflections from an intervention in eGovernment. European Journal of Information Systems, 21(2).

Park, J.-G., \& Lee, J. (2014). Knowledge sharing in information systems development projects: Explicating the role of dependence and trust. International Journal of Project Management, 32(1), 153-165.

Patton, M. (2015). Qualitative Research and Evaluation Methods. SAGE Publications, Inc; Fourth Edition edition. 11.

Pemsel, S., \& Wiewiora, A. (2013). Project management office a knowledge broker in project based organisations. International Journal of Project Management, 31(1), 31-42.

Pournader, M., Tabassi, A. A., \& Baloh, P. (2015). A three-step design science approach to develop a novel human resource-planning framework in projects: the cases of construction projects in USA, Europe, and Iran. International Journal of Project Management, 33(2), 419-434.

Reed, A. H., \& Knight, L. V. (2010). Effect of a virtual project team environment on communicationrelated project risk. International Journal of Project Management, 28(5), 422-427.

Rosa, D. V., Chaves, M. S., Oliveira, M., \& Pedron, C. (2016). Target: A Collaborative Model based on Social Media to Support the Management of Lessons Learned in Projects. International Journal of Managing Projects in Business. 4.

Turban, E., Liang, T. P., \& Wu, S. P. J. (2011). A Framework for Adopting Collaboration 2.0 Tools for Virtual Group Decision Making. Group Decision and Negotiation, 20(2), 137-154.

Veronese, G. T. \& Chaves, M. S. (2016). An Integrated Approach to Collaborative Learning in Projects. Strategic Integration of Social Media into Project Management Practice. IGI Global.

Wieringa, R. (2012). Technical Action Research as a Validation. In Proceeding DESRIST'12 Proceedings of the 7th international conference on Design Science Research in Information Systems: advances in theory and practice. 220-238.

Wiewiora, A., \& Murphy, G. (2015). Unpacking lessons learned": investigating failures and considering alternative solutions. Knowledge Management Research \& Practice, 13(1), 17-30.

Paper submitted

Paper accepted for publishing

Paper published online
O6 September 2019

11 November 2019

30 November 2019 